\title{
Regularized Adversarial Training (RAT) for Robust Cellular Electron Cryo Tomograms Classification
}

\author{
Xindi Wu \\ Computational Biology Department \\ Carnegie Mellon University \\ Pittsburgh, USA \\ xindiw@andrew.cmu.edu
}

\author{
Haohan Wang \\ Language Technologies Institute \\ Carnegie Mellon University \\ Pittsburgh, USA \\ haohanw@cs.cmu.edu
}

\author{
Xiangrui Zeng \\ Computational Biology Department \\ Carnegie Mellon University \\ Pittsburgh, USA \\ xiangruz@andrew.cmu.edu \\ Eric P. Xing \\ Machine Learning Department \\ Carnegie Mellon University \\ Pittsburgh, USA \\ epxing@cs.cmu.edu
}

\author{
Xin Gao \\ Computational Bioscience Research Center \\ King Abdullah University of Science and Technology \\ Thuwal, Saudi Arabia \\ xin.gao@kaust.edu.sa \\ $\operatorname{Min} \mathrm{Xu}^{+}$ \\ Computational Biology Department \\ Carnegie Mellon University \\ Pittsburgh, USA \\ mxu1@cs.cmu.edu
}

\begin{abstract}
Cellular Electron Cryo Tomography (CECT) 3D imaging has permitted biomedical community to study macromolecule structures inside single cells with deep learning approaches. Many deep learning-based methods have since been developed to classify macromolecule structures from tomograms with high accuracy. However, several recent studies have demonstrated the lack of robustness in these models against oftenimperceptible, designed changes of input. Therefore, making existing subtomogram-classification models robust remains a serious challenge. In this paper, we study the robustness of the state-of-the-art subtomogram classifier on CECT images and propose a method called Regularized Adversarial Training (RAT) to defend the classifier against a wide range of designed threats. Our results show that RAT improves robustness for CECT image classification over the previous methods.
\end{abstract}

Index Terms-Cellular Electron Cryo Tomography, Classification, Robustness, Adversarial Training, Adversarial Attacks.

\section{INTRODUCTION}

A precise understanding of the structure and spatial organization of all macromolecules inside single cells is an essential step towards understanding macromolecule-governed cellular processes. Cellular Electron Cryo Tomography (CECT) [1], as an emerging technique, provides three-dimensional views of

+ Correspoinding author.

This work was supported in part by U.S. National Institutes of Health (NIH) grant P41 GM103712. HW was supported by the National Institutes of Health grants R01-GM093156 and P30-DA035778. XZ was supported by a fellowship from Carnegie Mellon University's Center for Machine Learning and Health. XG was supported by the King Abdullah University of Science and Technology (KAUST) Office of Sponsored Research (OSR) under Award No. BAS/1/1624, FCC/1/1976-18, FCC/1/1976-23, FCC/1/197625, and FCC/1/1976-26. macromolecules inside single cells in their native, frozen, hydrated state, thus, leading to an unprecedented era of applying statistical methods for systematic discovery of macromolecular structures. There are plenty of previous works devoted to separating structurally highly heterogeneous macromolecules captured by CECT data into structurally homogeneous subgroups, such as unsupervised subtomogram ${ }^{1}[2]-[6]$ and deep learning based approaches for classification [7], [8].

Deep learning has become one of the most dominant classification methods, exhibiting impressive predictive performance in a variety of different biomedical applications [9]. Researchers nonetheless noticed an unsettling property of these deep models: a small perturbation of the sample may alter the prediction of a model dramatically [10], [11]. These perturbed examples are known as adversarial examples and the differences between original samples and adversarial examples may not even be perceivable to human [12]. This known vulnerability of neural networks may post serious robustness challenges of deep learning models in biomedical areas [13], [14], which naturally leads the follow-up questions such as whether these issues may affect the methods used on CECT data and how we can mitigate these issues if they exist.

In this paper, we first verify the vulnerability of the stateof-the-art deep learning models used on CECT data, then we propose a new efficient defense method called Regularized Adversarial Training (RAT) to improve the robustness of

\footnotetext{
${ }^{1}$ A subtomogram is a small cubic sub-image of a tomogram that contains a single macromolecule.
} 
classifiers against adversarial examples, which the models have no knowledge of. Our experimental results show that our method improves robustness on an existing state-of-the-art subtomogram classification model and is consistently effective against a variety of differently generated adversarial examples. We compare our method with existing defense methods and observe the model trained with our method performs much better in both accuracy and robustness. In addition, we also explore how the regularization parameter in our method affects the performance of the model.

Our main contributions can be summarized as follows:

- We test the robustness of the current state-of-the-art deep learning methods for subtomogram classification, and reveal the vulnerability against adversarial examples.

- We propose a new method called Regularized Adversarial Training (RAT) that it can push the decision boundary, which is made by model from original training sample and adversarial training sample, away from the data while maximizing the natural accuracy during training.

- We show that our proposed method improves the robustness over the state-of-the-art model on subtomogram classification.

\section{RELATED WORK}

For a discussion of related work, we first introduce previous work for subtomogram classification; then, we briefly discuss related challenges in building robust models for biomedical applications; finally, we summarize the related topics for improving the adversarial robustness of general-purpose deep learning models, offering context for follow-up sections.

Subtomogram Classification Recently, unsupervised classification approaches for subtomograms have been developed [2], [3], [6] but have strictly limited computational scalability. In the work of [8], deep learning based methods have been applied to CECT images, and large-scale 3D subtomogram classification models such as DSRF3D-v2, RB3D, and CB3D have achieved solid results on subtomograms. However, we show that after applying adversarial attacks to cell images, they achieve only low classification accuracy.

Robust Biomedical Classification While deep learning has helped the models achieve unprecedentedly high prediction accuracy, the high-reliability requirement of biomedical application have created new challenges [15]. In particular, the heterogeneous nature of the biomedical data misleads the model to learn confounding factors rather than genuine signals [9]. Several solutions have been proposed to calibrate the learning process for deep learning methods in biomedical applications [16] or general vision applications [17], [18]. However, recent works have revealed the vulnerability of biomedical deep learning models towards delicately crafted adversarial examples [13], [14].

Adversarial Robustness of Deep Learning Ever since the discovery of adversarial examples [10], [11], the related research has progressed as a race between two groups: one group of researchers aims to deceive the model and invented powerful methods (known as attack methods) such as FGSM [11],
DeepFool [19], and many others [12], [20]-[24], while the other group aims to defend the model against the deception and proposed a wide range of methods (known as defense methods) [25]-[29]. For instance, Adversarial training [30] increases robustness using data augmentation for training data with adversarial examples. TRADES loss [31], which introduces regularization to loss function to push the decision boundary away from samples is another popular strategy to achieve robust learning system.

\section{METHOD}

In this section, we discuss the notation and current state-ofthe-art model before introducing our proposed method.

\section{A. Notation}

Consider our subtomogram classification problem with the training set $\left\{\mathbf{x}_{i}, \mathbf{y}_{i}\right\}_{i=1}^{N}$ sampled from the distribution $\mathcal{D}$, where $\mathbf{x}_{i}$ denotes the $i$-th sample from subtomograms extracted from CECT images, $\mathbf{y}_{i} \in\{1, \ldots K\}$ is the label, $N$ denotes the number of samples and $K$ denotes the categories of labels. The network structure with parameters $\theta \in \mathbb{R}^{p}$ is denoted as $f(\mathbf{x} ; \theta)$, and $f_{l}(\mathbf{x} ; \theta)$ denotes the network $f(\mathbf{x} ; \theta)$ but outputs the softmax instead of prediction. The loss function (e.g. cross-entropy) of the model on $(\mathbf{x}, \mathbf{y})$ corresponds to $\mathcal{L}(f(\mathbf{x} ; \theta), \mathbf{y})$. A small adversarial perturbation is denoted by $\epsilon \in \mathbb{R}^{d}$. The adversarial sample is defined as $\mathbf{x}_{a d v}$, while the perturbation is $\epsilon_{1}=\mathbf{x}_{a d v}-\mathbf{x}$ and $\epsilon_{1} \in u_{i}$, where $u_{i}$ represents the neighborhood of $\mathbf{x}$. Similarly, the perturbed adversarial sample generated by adding label-free perturbation to adversarial sample is defined as $\mathbf{x}_{a d v}^{\prime}$, while the perturbation is $\epsilon_{2}=\mathbf{x}^{\prime}{ }_{a d v}-\mathbf{x}_{a d v}$ and $\epsilon_{2} \in u_{i}^{\prime}$, where $u_{i}^{\prime}$ represents the neighborhood of $\mathbf{x}_{a d v}$.. The $\ell_{p}$ norm for $1 \leq p<\infty$ of a vector $\mathbf{x}$ is denoted to be $\|\mathbf{x}\|_{p}=\left(\sum_{j=1}^{d}\left|\mathbf{x}_{i}\right|^{p}\right)^{1 / p}$ and the $\ell_{\infty}$ norm is $\|\mathbf{x}\|_{\infty}=\max _{i}\left\{\left|\mathbf{x}_{i}\right|\right\} . \nabla_{\mathbf{x}} g(\mathbf{x}, \mathbf{y})$ is denoted to be the gradient of $g$ with respect to the vector $\mathbf{x}$.

\section{B. Convolution-based 3D Classification Model}

Convolution-based 3D classification model (CB3D) [8] was proposed to achieve state-of-the-art deep learning based subtomogram classification performance. $3 \mathrm{D} \mathrm{CNN}$ was used due to the structures of these macromolecules. We adopt CB3D model as the basic classifier module in our experiments. The CB3D network contains eight layers. The first two layers both have one 3 D $3 \times 3 \times 3$ convolution layer with max pooling layer. The next three layers contain two $3 \mathrm{D} 3 \times 3 \times 3$ convolution layers with max pooling layer. The next two layers are fully connected layers and a softmax activation is appended to calculate the probabilities of each class. ReLU is applied as the activation function for all these layers except the last layer.

\section{Regularized Adversarial Training (RAT)}

Considerable efforts have been devoted to defense against adversarial samples, such as TRADES loss [31]. TRADES loss defines a loss function that contains two term: the natural loss encouraging the improvement of accuracy, and the robust loss encouraging the amelioration of robustness. However, directly 


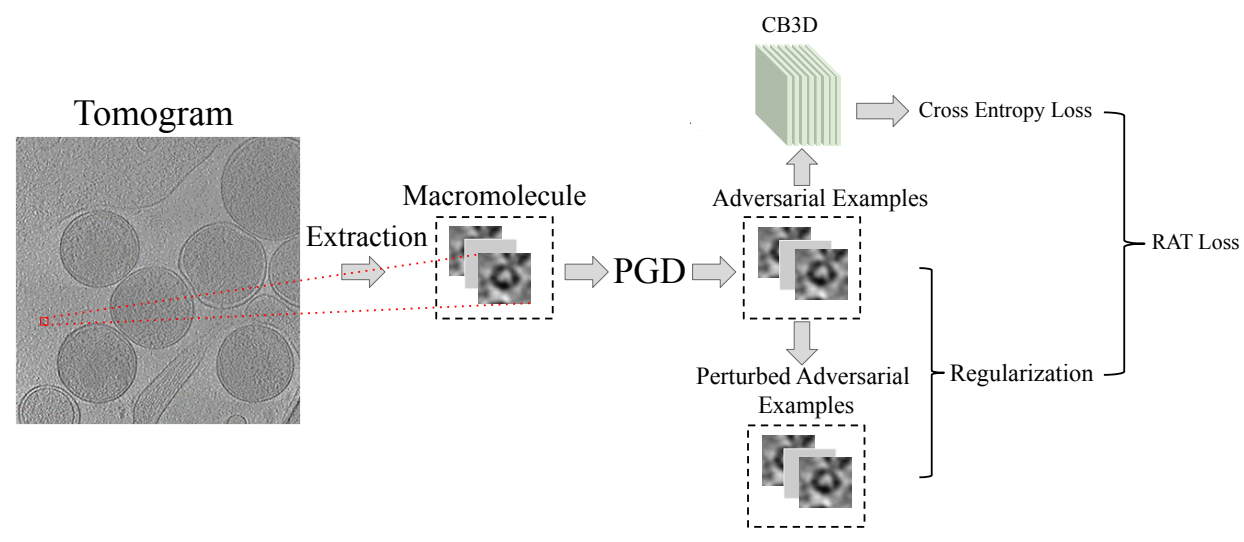

Fig. 1. A conceptual diagram of adversarial training subtomogram classification architecture. Note that those 2D-slice images are on behalf of 3D subtomograms. Also, the noise pattern is magnified 50 times in this picture in order to view it clearly in this conceptual diagram. This tomogram is from [32]

adopting TRADES loss seems not powerful enough to improve the robustness of the network as the effect of robust loss term, which can be regarded as a regularization, is limited by the requirement of accuracy and still faces the threat of overfitting. In order to make our model robust against various threats in addition to a wide variety of menacing attacks, inspired by adversarial training and TRADES loss, we propose a new defense method called Regularized Adversarial Training (RAT) which combines adversarial training and regularized discrepancy minimization to perform both high accuracy and strong robustness. Concretely, our method is in the form of

$$
\begin{gathered}
\min \mathbb{E}_{(\mathbf{x}, \mathbf{y}) \sim \mathcal{D}}\left(\operatorname { m a x } _ { \mathbf { x } _ { a d v } \in \mathbb { B } ( \mathbf { x } , \epsilon _ { 1 } ) } \left[\mathcal{L}\left(f\left(\mathbf{x}_{a d v} ; \theta\right) ; \mathbf{y}\right)+\right.\right. \\
\left.\left.\gamma_{\mathbf{x}^{\prime}{ }_{a d v} \in \mathbb{B}\left(\mathbf{x}_{a d v}, \epsilon_{2}\right)} k\left(f_{l}\left(\mathbf{x}^{\prime}{ }_{a d v} ; \theta\right), f_{l}\left(\mathbf{x}_{a d v} ; \theta\right)\right)\right]\right),
\end{gathered}
$$

where $k(\cdot, \cdot)$ is distance function and $\gamma>0$ is the regularization parameter.

The first term in 1 mainly comes from adversarial training. The main idea of adversarial training is to increase the model's robustness using adversarial examples as the training data [33]-[35] The classification model is trained on the adversarial distribution generated by projected gradient descent.

Following the most popular scheme, we use this objective:

$\min _{\theta} \mathcal{L}(f(\mathbf{x} ; \theta), \mathbf{y})=\mathbb{E}_{(\mathbf{x}, \mathbf{y}) \sim \mathcal{D}} \min _{\theta}\left[\max _{\epsilon_{1} \in u_{i}} \mathcal{L}\left(f\left(\mathbf{x}+\epsilon_{1} ; \theta\right), \mathbf{y}\right)\right]$,

to formulate the aim of adversarial training. This objective function acts as an upper bound of the robust error. The main idea is to increase model's robustness by ensuring the model will predict the same class for perturbed examples as well as clean images. To be specific, we use Projected Gradient Descent (PGD) [23] to generate the perturbed examples.

For each batch of natural examples $\left\{\mathbf{x}_{1}, \ldots, \mathbf{x}_{m}\right\}$, it starts from a random perturbation around the examples in a $l^{\infty}$ ball around a data example as a seed and follows the gradient of the current model weights regarding the input to generate a set of adversarial images $\left\{\mathbf{x}_{a d v 1}, \ldots, \mathbf{x}_{a d v m}\right\}$ in each training iteration. The generation process comprises of several update process. The update rule can be formed as:

$$
\begin{aligned}
\mathbf{x}_{a d v_{l+1}}= & \operatorname{Clip}_{[a, b]}\left\{\mathbf{x}_{a d v_{l}}+\right. \\
& \left.\mu_{1} \operatorname{sign}\left(\nabla_{\mathbf{x}_{a d v_{l}}} \mathcal{L}\left(f\left(\mathbf{x}_{a d v_{l}}, \theta\right), \mathbf{y}\right)\right)\right\},
\end{aligned}
$$

where $\mathbf{x}_{a d v_{l}}$ represents the adversarial samples after $l$-th update, $\mu_{1}$ is the step size of perturbation in each update to form the adversarial examples and the outer clip function Clip $_{[a, b]}$ keeps $\mathbf{x}_{a d v_{l}}$ in a perturbed range defined in advance. In general, it can be interpreted as an iterative algorithm to solve the following problem:

$$
\max _{\mathbf{x}_{a d v} \in \mathbb{B}\left(\mathbf{x}, \epsilon_{1}\right)} \mathcal{L}\left(f\left(\mathbf{x}_{a d v} ; \theta\right), \mathbf{y}\right) .
$$

To train a more robust model, we define a regularization measuring the discrepancy of decisions made by the model from adversarial examples,shown as the second term in 1 . The primary concern is to minimizing the distance between the predictions of adversarial sample $\mathbf{x}_{a d v}$ and the predictions of sample $\mathbf{x}^{\prime}{ }_{a d v}$ generated from $\mathbf{x}_{a d v}$ by a label-free algorithm. Like 4, the $\mathbf{x}_{a d v}^{\prime}$ is generated by solving the maximization problem:

$$
\max _{\mathbf{x}^{\prime}{ }_{a d v} \in \mathbb{B}\left(\mathbf{x}_{a d v}, \epsilon_{2}\right)} k\left(f_{l}\left(\mathbf{x}^{\prime}{ }_{a d v} ; \theta\right), f_{l}\left(\mathbf{x}_{a d v} ; \theta\right)\right) .
$$

This algorithm is iterative and the update rule is like 3 , which in $l$-th update is formulated as:

$$
\begin{aligned}
\mathbf{x}^{\prime}{ }_{a d v l+1}= & \operatorname{Clip}_{\left[a^{\prime}, b^{\prime}\right]}\left\{\mathbf{x}^{\prime}{ }_{a d v_{l}}+\right. \\
& \mu_{2} \operatorname{sign}\left(\nabla_{\mathbf{x}^{\prime}{ }_{a d v_{l}}} k\left(f_{l}\left(\mathbf{x}^{\prime}{ }_{a d v l} ; \theta\right), f_{l}\left(\mathbf{x}_{a d v} ; \theta\right)\right)\right\},
\end{aligned}
$$

where $\mathbf{x}_{a d v l}^{\prime}$ represents the perturbed adversarial samples generated from $\mathbf{x}_{a d v}$ after $l$-th update.

Here we use KullbackLeiblerdivergence [36] to measure the distance between $f_{l}\left(\mathbf{x}^{\prime}{ }_{a d v} ; \theta\right)$ and $f_{l}\left(\mathbf{x}_{a d v} ; \theta\right)$. The softmax outputs computed by $f_{l}(\cdot ; \cdot)$ are fed into the function as probability distribution. The regularization parameter $\gamma$ is 
critical of controlling the effect of regularization. The method tends to original adversarial training when $\gamma \rightarrow 0$ as the effort of regularization is almost eliminated, and tends to the all-one classifier when $\gamma \rightarrow+\infty$ as the effort of regularization almost dominates during optimization.

The regularization can be somehow regarded as an unsupervised process because it does not depend on label $\mathbf{y}$. It first perturbs the adversarial samples only depending on adversarial sample by maximizing the "difference" between label determining adversarial samples $\mathbf{x}_{a d v}$ and data determining adversarial samples $\mathbf{x}^{\prime}{ }_{a d v}$.

By optimizing the network to get the similar prediction from $\mathbf{x}_{a d v}$ and $\mathbf{x}_{a d v}^{\prime}$ in which the "difference" is large enough, the network can learn the robust features and ignore the easily been influenced features or features introduced by adversarial training from given images. Intuitively, since adversarial training can be viewed as the training set extension and augmentation and the first term in 1 can be regarded as minimizing the "distance" between $f(\mathbf{x} ; \theta)$ and $\mathbf{y}$ while improving robustness, our method regularizes the adversarial training process by minimizing the "distance" between the prediction of $\mathbf{x}_{a d v}$ and prediction of $\mathbf{x}^{\prime}{ }_{a d v}$ to push the decision boundary of network away from the adversarial samples fed in the network, improving network robustness.

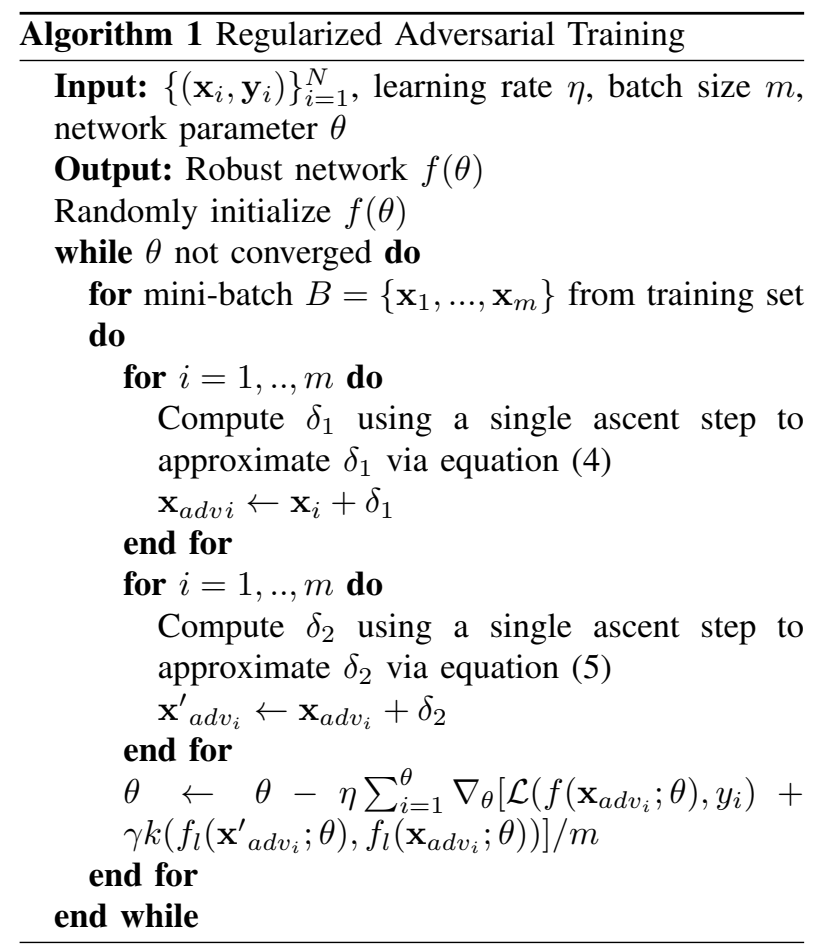

IV. EXPERIMENT

\section{A. Dataset}

We use the dataset [37] which includes 7 structural classes, each with 400 subtomograms from EMPIAR. The SNR is 0.5 and missing wedge angle is 30 degrees. Each particle is consisted of $28^{3}$ voxels, and the size of each voxel is $0.94 \mathrm{~nm}$. For each tomogram downloaded from EMPIAR, we extracted potential structural regions using Difference of Gaussian with parameters $s 1=7.0$ and $k=1.1$. Subvolumes of size $28 \times 28 \times 28$ were extracted and about 20 macromolecules were manually picked. The 20 subtomograms were averaged to generate the structural template. Structural template was aligned to all subvolumes extracted and produces crosscorrelation scores. The top 1000 subvolumes were selected and we manually identified 400 subtomograms for each class.

\section{B. Training Details}

Our proposed model has been trained on a system with an NVIDIA GTX 1080 GPU for 100 epochs and the batch size is 16. We use TensorFlow to implement the proposed model. The entire dataset contains 2800 subtomograms. $80 \%$ images are sampled as the training set and the remaining ones are used as testing samples. We do not consider a validation set due to the small sample size. For every batch of data during training, we follow Equation 3 to perturb the original images. Both $[a, b]$ and $\left[a^{\prime}, b^{\prime}\right]$ are set as $[-0.01,0.01] . \epsilon$ is set as 0.01 and step size $\mu$ is set to 0.001 for both $\mathbf{x}_{a d v}$ and $\mathbf{x}^{\prime}{ }_{a d v}$.

Consistent with the implementation in [30], we use both 10 steps to generate adversarial images $\mathbf{x}_{a d v}$ and perturbed adversarial images $\mathbf{x}_{a d v}^{\prime}$. The hyperparameter $\gamma$ is set to 1 for the attack against CB3D and attack against robustly trained CB3D experiments. We use cross-entropy as loss function at the first term in 1 and use ADAM for optimizing the parameters. The initial learning rate is 0.0001 .

Besides, we also apply several techniques from recent works to stabilize the training procedure. Zero-mean normalization is applied to normalize both the training set and testing set. Dropout [38] is applied for reducing overfitting in neural network and the rate is set to 0.5 .

\section{Construction of adversarial attacks and defenses}

We start the experiments to attack the state-of-the-art model and test the effectiveness of our defense method against a wide range of attack methods and compare with other methods, i.e. adversarial training and TRADES loss. The adversarial attack methods we used here include:

- Fast Gradient Sign Attack (FGSM) [11] considers the sign of the gradient of the model and perturbs the images along it to increase the prediction loss. We control the $\max \epsilon$ of perturbation to control the performance.

- DeepFool $L 2 / L \infty$ Attack [19] approximates the model with a linear classifier and finds the closest projection of the original image onto the space of misclassification.

- Carlini and Wagner Attack [21] searches for the adversarial images that the disparity between these images and raw images is as small as possible while the adversarial samples are misclassified.

- Additive Uniform Noise Attack [39] The standard deviation of noise gradually increases until misclassified.

- Salt and Pepper Noise Attack [39] repeatedly adds the salt and pepper noise to the image until misclassified.

We also compare adversarial training and TRADES loss to demonstrate the efficiency of our method. We first apply attack against CB3D that we use the attack methods mentioned above 
to attack the natural training CB3D network and generate the adversarial images. These attack methods are implemented using foolbox. We apply FGSM attack here with setting max $\epsilon$ to 0.03 . We load different models and evaluate them on these adversarial images. The results are shown in Table I.

Furthermore, we verify the performance of our method against adversarial examples of robustly trained CB3D. We apply FGSM method to attack models training with our method, adversarial training and TRADES loss. Additive Uniform Noise Attack and Salt and Pepper Noise Attack are not applied because they are decision-based attacks [40] requiring neither gradients nor probabilities. To make our conclusion more convincing, we apply FGSM with setting max $\epsilon$ to 0.03 , 0.06 and 0.12 . The results are illustrated in Table II.

\section{RESUlts AND Discussions}

\section{A. Against Adversarial Examples of $C B 3 D$}

We reported the experimental results in Table I. For the results that the adversarial images coming from natural training model, the vulnerability of existing state-of-art model for subtomogram classification can be revealed since the accuracy of natural training model is extremely low under all kind of attack methods. Then we can see our method makes a enormous progress to defend all these attack methods, especially on FGSM, DeepFool $L 2$, DeepFool $L \infty$ and CW. All of them achieve high accuracy results (over 90\%). The accuracy of model training with our method on Additive Uniform Noise Attack and Salt\&Pepper Noise Attack is not as good as others. This is mainly because that these two attack methods are decision-based attacks, which are direct attacks that solely depend on the final decision made by the model, not the gradient. Thus, the gradient-based defense methods, e.g. ours, adversarial training and TRADES loss, are hard to optimize network to get the outstanding performance, though our proposed method still retains its dominant position. The defend performances of our method are much better than the other two methods on FGSM and Salt\&Pepper Noise Attack methods. However, when applying Additive Uniform Noise Attack, the adversarial training method achieves the best result in the three methods. We can speculate that it is because of the regularization. The regularization aims to minimize the difference between adversarial images and its original images from the network perspective based on gradient. So when it comes to Uniform Noise, which randomly adds a stochastic number to every voxel, regularization is not embodied as the "difference" between adversarial images with uniform noise and original images is similar with the "difference" in regularization. Unlike uniform noise, Salt\&Pepper Noise Attack method only randomly modifies part of voxel to either the maximum value or minimum value of the images, avoiding obscuring the effect of regularization.

\section{B. Against Adversarial Examples of Robustly Trained CB3D}

We summarized our results against adversarial examples of robustly trained CB3D in Table II. Although the accuracy on all attacks drops significantly from the natural accuracy, which is result of evaluating on natural images, the model with our method still achieve the best results among these models. While applying FGSM attack method, the accuracy of our method is all more than $20 \%$ and the accuracy of other defense method is extremely low, which seems that the network randomly classifies the adversarial images completely.

\section{CONCLUSION}

In this paper, we studied the robustness of the state-ofthe-art deep learning model on CECT images (i.e. the CB3D model). We first verified the existing vulnerability of the model and then developed Regularized Adversarial Training (RAT) to improve the robustness of $\mathrm{CB} 3 \mathrm{D}$ for one of the pioneering robust biomedical image classification models against adversarial corruptions. We experimented with a wide range of adversarial attack methods. Experimental results demonstrated that Regularized Adversarial Training can significantly improve robustness of the deep learning based subtomogram classification against a wide range of adversarial attacks effectively. This work represents a meaningful step towards robust classification and recovery of millions of macromolecules extracted from CECT images. We hope our method can help the biomedical community improve robustness of other biomedical image classification methods against adversarial attacks.

\section{REFERENCES}

[1] S. Asano, B. D. Engel, and W. Baumeister, "In situ cryo-electron tomography: a post-reductionist approach to structural biology," Journal of molecular biology, vol. 428, no. 2, pp. 332-343, 2016.

[2] X. Chen, Y. Chen, J. M. Schuller, N. Navab, and F. Förster, "Automatic particle picking and multi-class classification in cryo-electron tomograms," in 2014 IEEE 11th International Symposium on Biomedical Imaging (ISBI), pp. 838-841, IEEE, 2014.

[3] M. Xu, M. Beck, and F. Alber, "High-throughput subtomogram alignment and classification by fourier space constrained fast volumetric matching," Journal of structural biology, vol. 178, no. 2, pp. 152-164, 2012.

[4] M. Xu and F. Alber, "High precision alignment of cryo-electron subtomograms through gradient-based parallel optimization," BMC systems biology, vol. 6, no. 1, p. S18, 2012.

[5] M. Xu and F. Alber, "Gradient-based high precision alignment of cryoelectron subtomograms," in 2011 IEEE International Conference on Systems Biology (ISB), pp. 279-284, IEEE, 2011.

[6] T. A. Bharat, C. J. Russo, J. Löwe, L. A. Passmore, and S. H Scheres, "Advances in single-particle electron cryomicroscopy structure determination applied to sub-tomogram averaging," Structure, vol. 23, no. 9 , pp. $1743-1753,2015$.

[7] M. Xu, X. Chai, H. Muthakana, X. Liang, G. Yang, T. Zeev-BenMordehai, and E. P. Xing, "Deep learning-based subdivision approach for large scale macromolecules structure recovery from electron cryo tomograms," Bioinformatics, vol. 33, no. 14, pp. i13-i22, 2017.

[8] C. Che, R. Lin, X. Zeng, K. Elmaaroufi, J. Galeotti, and M. Xu, "Improved deep learning-based macromolecules structure classification from electron cryo-tomograms," Machine Vision and Applications, vol. 29, no. 8, pp. 1227-1236, 2018.

[9] T. Yue and H. Wang, "Deep learning for genomics: A concise overview," arXiv preprint arXiv:1802.00810, 2018.

[10] C. Szegedy, W. Zaremba, I. Sutskever, J. Bruna, D. Erhan, I. Goodfellow, and R. Fergus, "Intriguing properties of neural networks," arXiv preprint arXiv:1312.6199, 2013.

[11] I. J. Goodfellow, J. Shlens, and C. Szegedy, "Explaining and harnessing adversarial examples (2014)," in International Conference on Learning Representations, 2015.

[12] H. Wang, X. Wu, P. Yin, and E. P. Xing, "High frequency component helps explain the generalization of convolutional neural networks," arXiv preprint arXiv:1905.13545, 2019. 
TABLE I

Against AdVERSARIAL EXAMPLES OF CB3D

\begin{tabular}{|c|c|c|c|c|c|c|c|c|}
\hline \multirow{2}{*}{$\begin{array}{c}\text { Defense } \\
\text { Model }\end{array}$} & Attacked & Natural & \multicolumn{5}{|c|}{ Robust Accuracy } \\
\cline { 6 - 9 } & Model & Accuracy & FGSM $^{0.03}$ & DeepFool $L_{2}$ & DeepFool $L_{\infty}$ & CW & Salt\&Pepper & Noise \\
\hline Natural training & Natural training & 97.32 & 14.29 & 0.36 & 0.18 & 0.00 & 1.96 \\
\hline Adversarial Training & Natural training & 98.39 & 89.28 & 95.53 & 93.75 & 86.25 & 46.07 & 36.96 \\
\hline TRADES loss & Natural training & 98.39 & 87.86 & 72.32 & 67.14 & 45.53 & 38.04 & 23.21 \\
\hline RAT & Natural training & 99.46 & 96.07 & 97.32 & 97.86 & 90.18 & 70.54 & 23.21 \\
\hline
\end{tabular}

TABLE II

AgAinst AdVERSARIAl EXAMPLES OF Robustly TRAINED CB3D

\begin{tabular}{|c|c|c|c|c|}
\hline Defense Model & Under which Attack & $\mathbf{L} \infty$ Distance $^{\perp}$ & Natural Accuracy (\%) $^{\text {Robust Accuracy (\%) }}$ \\
\hline Natural training & & 0.0356 & 97.32 & 14.29 \\
Adversarial Training & \multirow{2}{*}{ FGSM $^{0.03}$} & 0.0513 & 98.39 & 14.29 \\
TRADES loss & & 0.0348 & 98.39 & 15.18 \\
RAT & & $\mathbf{0 . 0 6 8 6}$ & $\mathbf{9 9 . 4 6}$ & $\mathbf{2 7 . 5 0}$ \\
\hline Natural training & & 0.0358 & 97.32 & 14.29 \\
Adversarial Training & \multirow{2}{*}{ FGSM $^{0.06}$} & 0.0515 & 98.39 & 14.29 \\
TRADES loss & & 0.0414 & 98.39 & 14.46 \\
RAT & & $\mathbf{0 . 0 7 0 5}$ & $\mathbf{9 9 . 4 6}$ & $\mathbf{2 7 . 3 2}$ \\
\hline Natural training & & 0.0359 & 97.32 & 14.29 \\
Adversarial Training & \multirow{2}{*}{ FGSM $^{0.12}$} & 0.0518 & 98.39 & 14.46 \\
TRADES loss & & 0.0416 & 98.39 & $\mathbf{2 7 . 3 2}$ \\
RAT & & $\mathbf{0 . 0 7 0 8}$ & $\mathbf{9 9 . 4 6}$ & \\
\hline
\end{tabular}

${ }^{1}$ The average $\mathrm{L} \infty$ distance of all images.

[13] S. G. Finlayson, H. W. Chung, I. S. Kohane, and A. L. Beam, "Adversarial attacks against medical deep learning systems," arXiv preprint arXiv:1804.05296, 2018.

[14] Y. Huang, T. Würfl, K. Breininger, L. Liu, G. Lauritsch, and A. Maier, "Some investigations on robustness of deep learning in limited angle tomography," in International Conference on Medical Image Computing and Computer-Assisted Intervention, pp. 145-153, Springer, 2018.

[15] R. Miotto, F. Wang, S. Wang, X. Jiang, and J. T. Dudley, "Deep learning for healthcare: review, opportunities and challenges," Briefings in bioinformatics, vol. 19, no. 6, pp. 1236-1246, 2017.

[16] H. Wang, Z. Wu, and E. P. Xing, "Removing confounding factors associated weights in deep neural networks improves the prediction accuracy for healthcare applications," in Pacific Symposium on Biocomputing, 2019.

[17] H. Wang, A. Meghawat, L.-P. Morency, and E. P. Xing, "Select-additive learning: Improving generalization in multimodal sentiment analysis," 2017 IEEE International Conference on Multimedia and Expo (ICME), Jul 2017.

[18] H. Wang, Z. He, and E. P. Xing, "Learning robust representations by projecting superficial statistics out," in International Conference on Learning Representations, 2019.

[19] S.-M. Moosavi-Dezfooli, A. Fawzi, and P. Frossard, "Deepfool: a simple and accurate method to fool deep neural networks," in Proceedings of the IEEE conference on computer vision and pattern recognition, pp. 2574 2582, 2016.

[20] C. Xiao, B. Li, J.-Y. Zhu, W. He, M. Liu, and D. Song, "Generating adversarial examples with adversarial networks," arXiv preprint arXiv:1801.02610, 2018.

[21] N. Carlini and D. Wagner, "Towards evaluating the robustness of neural networks," in 2017 IEEE Symposium on Security and Privacy (SP), pp. 39-57, IEEE, 2017.

[22] J. Su, D. V. Vargas, and K. Sakurai, "One pixel attack for fooling deep neural networks," IEEE Transactions on Evolutionary Computation, 2019.

[23] A. Kurakin, I. Goodfellow, and S. Bengio, "Adversarial machine learning at scale," arXiv preprint arXiv:1611.01236, 2016.

[24] X. Chen, C. Liu, B. Li, K. Lu, and D. Song, "Targeted backdoor attacks on deep learning systems using data poisoning," arXiv preprint arXiv:1712.05526, 2017.

[25] N. Akhtar, J. Liu, and A. Mian, "Defense against universal adversarial perturbations," in Proceedings of the IEEE Conference on Computer Vision and Pattern Recognition, pp. 3389-3398, 2018.
[26] H. Lee, S. Han, and J. Lee, "Generative adversarial trainer: Defense to adversarial perturbations with gan," arXiv preprint arXiv:1705.03387, 2017.

[27] D. Meng and H. Chen, "Magnet: a two-pronged defense against adversarial examples," in Proceedings of the 2017 ACM SIGSAC Conference on Computer and Communications Security, pp. 135-147, ACM, 2017.

[28] J. H. Metzen, T. Genewein, V. Fischer, and B. Bischoff, "On detecting adversarial perturbations," arXiv preprint arXiv:1702.04267, 2017.

[29] W. He, J. Wei, X. Chen, N. Carlini, and D. Song, "Adversarial example defense: Ensembles of weak defenses are not strong," in 11th \{USENIX\} Workshop on Offensive Technologies (\{WOOT $\}$ 17), 2017.

[30] A. Madry, A. Makelov, L. Schmidt, D. Tsipras, and A. Vladu, "Towards deep learning models resistant to adversarial attacks," arXiv preprint arXiv:1706.06083, 2017.

[31] H. Zhang, Y. Yu, J. Jiao, E. P. Xing, L. E. Ghaoui, and M. I. Jordan, "Theoretically principled trade-off between robustness and accuracy," arXiv preprint arXiv:1901.08573, 2019.

[32] "Empiar-10048." https://www.ebi.ac.uk/pdbe/emdb/empiar/entry/10048/.

[33] I. J. Goodfellow, J. Shlens, and C. Szegedy, "Explaining and harnessing adversarial examples," arXiv preprint arXiv:1412.6572, 2014.

[34] C. Lyu, K. Huang, and H.-N. Liang, "A unified gradient regularization family for adversarial examples," in 2015 IEEE International Conference on Data Mining, pp. 301-309, IEEE, 2015.

[35] U. Shaham, Y. Yamada, and S. Negahban, "Understanding adversarial training: Increasing local stability of supervised models through robust optimization," Neurocomputing, vol. 307, pp. 195-204, 2018.

[36] S. Kullback and R. A. Leibler, "On information and sufficiency," The annals of mathematical statistics, vol. 22, no. 1, pp. 79-86, 1951.

[37] A. J. Noble, V. P. Dandey, H. Wei, J. Brasch, J. Chase, P. Acharya, Y. Z. Tan, Z. Zhang, L. Y. Kim, G. Scapin, et al., "Routine single particle cryoem sample and grid characterization by tomography," Elife, vol. 7, p. e34257, 2018.

[38] G. E. Hinton, N. Srivastava, A. Krizhevsky, I. Sutskever, and R. R. Salakhutdinov, "Improving neural networks by preventing co-adaptation of feature detectors," arXiv preprint arXiv:1207.0580, 2012.

[39] J. Rauber, W. Brendel, and M. Bethge, "Foolbox: A python toolbox to benchmark the robustness of machine learning models," arXiv preprint arXiv:1707.04131, 2017.

[40] W. Brendel, J. Rauber, and M. Bethge, "Decision-based adversarial attacks: Reliable attacks against black-box machine learning models," arXiv preprint arXiv:1712.04248, 2017. 